\title{
Pierre Menard y la edición académica en Bibliotecología y Ciencia de la Información
}

\section{Alejandro E. Parada}

\begin{abstract}
Secretario de redacción / Información, cultura y sociedad Universidad de Buenos Aires, Facultad de Filosofía y Letras, Instituto de Investigaciones Bibliotecológicas-INIBI, Argentina / aparada@filo.uba.ar / Orcid ID: https://orcid.org/oooo-ooo2-2331-442X
\end{abstract}

\section{Resumen}

En este editorial, con motivo de los veinte años de Información, Cultura y Sociedad, se analizan los problemas y los nuevos desafíos en la edición de las revistas académicas. Además se reflexiona sobre una serie de aspectos relacionados con los procesos editoriales comerciales y el acceso abierto. Finalmente, se abordan algunas propuestas preliminares sobre este tema.

\section{Palabras clave}

Edición de revistas académicas Revistas especializadas Bibliotecología y Ciencia de la Información

Aspectos teóricos

Pierre Menard and the academic edition in Librarianship and Information Science

\begin{abstract}
In this editorial, on the occasion of the twenty years of Informacion, Culturay Sociedad, the problems and the new challenges of the edition of academic journals are analyzed. It also reflects on a series of aspects related to commercial publishing processes and open access. Finally, some preliminary proposals on this subject are addressed.
\end{abstract}

Keywords

Academic Journal Publishing Academic Edition Librarianship and Information Science

Theorical aspects
Toda conmemoración corre el riesgo de caer en cierta idealización del pasado. De precipitarse en un matiz egocéntrico y en la trampa de pensarse dentro de la esfera de "lo imprescindible". Un lugar donde puede desplegarse lo bucólico (al fin de cuentas estamos en una Facultad de Humanidades) y algunas ensoñaciones románticas ante el desafío que hoy implica editar una revista en una universidad pública de América Latina, atravesada, inevitablemente, por el posmodernismo y las siempre constantes 
limitaciones económicas, aunque estas últimas suelen travestirse con un dudoso velo democrático y republicano. Información, Cultura y Sociedad, ahora definida por los aniversarios que la asedian, cumple, pues, 20 años de vida académica y presenta a sus lectores el número cuarenta.

No se trata de hacer un balance. Como en las antiguas narraciones o ensayos, "el curioso lector" puede recurrir a la totalidad de los números en su plataforma en línea (Revistas científicas Filo, 2018). Es posible así recorrer esas cuatro decenas para conocer su contenido y apelar al rigor de las estadísticas que bien pueden explicar muchos aspectos o, tal vez, simular la verdad. En el mundo de lo cuantitativo la libre interpretación y el encare subjetivo también es una forma de ficción. A nuestro criterio, en el presente editorial se manifiesta la oportunidad de realizar algunas reflexiones provisionales y no menos subjetivas. Reflexiones que pueden tomar la configuración de aspectos preliminares.

El primer aspecto que quisiera señalar es el temporal. Cuando en 1999 el Instituto de Investigaciones Bibliotecológicas de la Facultad de Filosofía y Letras de la Universidad de Buenos Aires comenzó con la publicación de Información, Cultura y Sociedad, el universo de la cultura impresa aún era indiscutible para las revistas científicas y, en particular, para las Humanidades y las Ciencias Sociales. Como es bien conocido, en el presente, la edición virtual y digital se ha empoderado casi por completo de las publicaciones académicas. Esto enfatiza el hecho de que estuvimos navegando en las corrientes de una de las mayores revoluciones editoriales. Esta modificación posee una complejidad que no solo se relaciona con los procedimientos de edición sino, principalmente, con las prácticas y representaciones de la lectura de las publicaciones periódicas especializadas. Dos décadas alcanzaron para dar por tierra con una tradición impresa (académica o no) que había comenzado en el siglo XVII cuando aparecieron las primeras revistas relacionadas con la ciencia.

Dicha transformación ha implicado cambios de edición y de lectura; por consiguiente, profundas mutaciones en las formas de escribir y de evaluar los artículos científicos. Lo que conlleva a rescatar un viejo concepto histórico: hemos sufrido una renovación copernicana en nuestra mentalidad de concebir las formas de producir un texto escrito. En veinte años, cuando la materialidad sobre el papel era contundente, se implementó una revolución de la inmaterialidad que dio vuelta con las tradiciones de la Cultura Escrita y superó, en gran medida, las intervenciones editoriales por parte de los editores que puntualizara McKenzie (2005) en el último tercio del siglo XX. En la actualidad, una revista especializada es uno de los mayores exponentes de las nuevas tecnologías virtuales de la Cultura Escrita, ahora eyectada hacia un destino abierto y, a veces, inquietante (El futuro..., 2019; CRECS, 2019). El aspecto temporal fue implacable y determinante con ese tipo de revistas y las reconvirtió completamente.

Otra característica en cuestión es la propiedad y sus derechos. En estos cuatro lustros la contienda entre la información como mercancía y su acceso abierto se desarrolló con un gran y voraz dinamismo. Las editoriales comerciales desplegaron su fortaleza para reconfigurar la literatura científica (el mejor modelo de un evolucionado "gatopardismo") en un bien de consumo del conocimiento, donde hay privilegiados onerosos y humildes entenados. Estas compañías afirmaron y consolidaron sus emprendimientos rentados, dictaminaron sobre los mecanismos de producción y de distribución, volvieron a conquistar a los investigadores de universidades públicas y privadas en el momento de pensar la publicación de sus trabajos en títulos internacionales, impusieron sus metodologías de evaluación y, ya hace mucho, comenzaron a asediar los fundamentos del acceso abierto. 
Consiste en una disyuntiva económica por parte de ámbito comercial, pero además desnuda nuestra complaciente autosumisión. Como toda controversia de fondo, se trata de un combate cultural. ¿Acaso no nos han seducido -como secretario de redacción de una revista, me incluyo- sobre el "factor impacto" en el momento de elegir una revista para editar un artículo ante el enriquecimiento de un conocimiento más compartido que plantean las recomendaciones de DORA (2013) y el Manifiesto de Leiden (Hicks et al, 2015) en los procesos de evaluación?

Los próximos años serán cruciales para anotarse algunos puntos en este debate entre una sociedad centrada en el capital y una circulación de la información con los menores obstáculos posibles. Incluso sería aconsejable volver a pensar filosófica y concretamente el tema siempre ríspido de aquello sobre lo qué es la propiedad de la informacióny, por extensión lógica, de los saberes. El ensayista estadounidense Bob Black (2017), acaso con una posición fundamentalista pero no menos interesante, plantea la necesidad de abolir el trabajo no solo porque resulta una servidumbre en la construcción social, sino porque genera una dependencia de la propiedad para su usufructo usurero. El acceso libre sería en este contexto un paso modesto pero vigoroso para abolir una de las tantas y disímiles formas del neocolonialismo informativo. Y un joven Karl Marx (1983 [1842]), mucho antes que pensara en escribir El Capital, defendía el derecho de los humildes a recoger la leña caída de los bosques que estaban bajo la esfera exclusiva de sus propietarios, asentando, en una elipsis no soñada para él, las bases para recoger libremente la información necesaria para subsistir y crecer, por derecho inajenable, en un sociedad del conocimiento.

Es necesario entonces reconquistar el territorio ocupado y establecer un predominio en nuestra propia gobernabilidad. Aquellos que estamos involucrados en la gestión de revistas científicas debemos renovar los procederes habituales de administrarlas como un objeto de mercado, por supuesto, en forma paulatina y con los mandatos académicos que exige cada entidad para validar los contenidos y sus criterios de verdad. Una muestra, en este punto, es ilustrativa: para superar los criterios de evaluación doble ciego por pares es condición necesaria, en particular, que cambien esos elementos de arbitraje en el epicentro mismo y en las normativas, en nuestro caso, de la Universidad de Buenos Aires.

Hay otros aspectos concluyentes que es oportuno abordar. Nos referimos al criterio de la construcción de los valores. Podríamos citar un ejemplo. El acceso libre, prototípico de los países en vías de desarrollo, alienta a los autores a publicar en sus plataformas pero, luego, los gestores comerciales cosechan en ellas y manipulan la esencia de su valor libre. Sobrevuela, pues, para muchos investigadores, el fantasma que, a la corta o a la larga, luego de alentarlos para que publiquen en acceso abierto, terminan también siendo cosechados por los mismos "tanques editoriales" de siempre. Y el término "fantasma" no es ocioso empleado en esta trama: Ivan Illich (2008) denominó "trabajo fantasma" a aquellas labores no remuneradas en la cual se basa y se apropia la sociedad industrial-capitalista como un complemento fundamental para sostener sus bienes y privilegios productivos, tal como operan las mega empresas transnacionales de edición científica.

Por otra parte, y dentro de esta temática, hay autores que plantean reflexiones axiológicas más profundas. El acceso libre se encuentra, de manera relativa, bien encaminado en América Latina, fundamentalmente en las Humanidades y las Ciencias Sociales, pero no es sólido en las ciencias duras. Esta situación plantea la posibilidad (de hecho, una realidad) de fomentar un cisma cada vez más pronunciado entre la expansión de las ciencias duras en detrimento de las Ciencias Sociales; es decir, como ya muchos lo han observado, los autores en temáticas de Ciencias Exactas tienen una tendencia mayor a publicar en ámbitos comerciales que libres. Nuevamente, se 
plantea el tópico de la controversia cultural vinculada a los procesos de globalización y de prestigio autoral, donde el "factor impacto" despliega su preponderancia.

Pero el tema valorativo, en la gestión de las publicaciones periódicas, posee particularidades todavía más profundas. Esto es visible en el criterio de deterioro de la identidad. Un criterio que hunde sus raíces en una concepción neopositivista de las ciencias en general. Veamos otro ejemplo que refuerza el concepto anterior y se imbrica, subrepticiamente, con las problemáticas de acceso libre. El portal de revistas de la Facultad de Filosofía y Letras de la Universidad de Buenos Aires, siendo una casa de estudios en Humanidades y Ciencias Sociales, se autodefine como "Revistas científicas de Filo" (2018). Lo que implica, al menos, una posición dubitativa acerca de su naturaleza humanística y social, y este es otro de los caminos, más sutiles, para desalentar otras formas de publicación, pues quien no reconoce su naturaleza difícilmente pueda defender plenamente sus derechos de acceso libre. Pero la crisis de identidad y los criterios de las ciencias duras en el ámbito de las Ciencias Sociales forman parte de la misma cara de una moneda, y ya nos hemos referido a este tema en otros editoriales.

También se potencia un nuevo y fascinante aspecto o categoría: el criterio de comunidad editorial. Un texto escrito que también es (y lo será cada vez más) modificable durante los procesos de edición; y lo que es más importante, un discurso que no estará definitivamente redactado y podrá (y deberá) ser modificado en forma permanente por sus autores y por la comunidad científica que lo aborda desde distintos ángulos y saberes. Esta revolución de veinte años desembocará, entonces, con probabilidad, en un texto latente y vivo, sin cierre final (o mejor, con sucesivos cierres finales): un tejido escrito en virtuosa expansión.

Nos encontramos, por lo tanto, ante una acelerada parcelación y fragmentación de aquello que se consideraba el artículo como unidad cerrada. Este nuevo y pujante reino de edición fragmentada trae como correlato la pérdida del criterio de individualidad (o trabajo en equipo) en la redacción de un trabajo, ya que los nuevos autores que modelarán nuestros próximos artículos volverán a rescatar algunos aspectos de los inicios de la escritura: el anonimato y el gesto coral-orquestal de variados autores.

No se trata, solo con exclusividad, de instrumentar las esferas ancladas en tecnologías virtuales, tales como marcar o etiquetar un artículo, o poseer (y manejarla a fondo) la última versión del OJS u otro software de código abierto en la gestión de revistas, o de difundir la matriz genética del Lenguaje de Marcado Extensible (XLM), que son fundamentales e imprescindibles sino, ante todo, de tomar conciencia de la esencia mudable del texto escrito que lo acerca a las características de un ente múltiple y poliforme, en una nueva etapa de la biología de la escritura y su distribución científica. Un desafío con tonalidades épicas pero ilimitadas en cuanto a la gestación de la escritura académica. El incremento de los preprints o la publicación previa "antes de publicar" denominada Online First cambiará el concepto de unidad cerrada de un número o volumen de una revista. De este modo, cada artículo en la web se convierte en un espacio de interacción recíproca donde su Digital Object Identifier (DOI) es capital para la identificación de los sucesivos cambios de un objeto digital en estos nuevos y transformadores procesos de publicación. Por supuesto, el desembolso económico del DOI, inevitablemente, es otra importante singularidad en esta discusión.

Aunque parezca paradójico Pierre Menard tiene mucho que ver con esta situación. No quisiera engrosar la redundante citación de Jorge Luis Borges; lo que importa, para nuestros intereses, es la riqueza del relato borgeano para reflexionar sobre los procedimientos de edición de una revista latinoamericana especializada en Bibliotecología y Ciencia de la Información (BCI). En este relato -hay casi infinitas interpretaciones- Borges es un verdadero precursor de la muerte del autor aún antes que 
lo planteara Roland Barthes y, además, de las múltiples escrituras que puede tener un mismo texto repetido en cada una de sus letras. Su personaje, el escritor francés Menard es capaz de apoderarse y citar "letra por letra" el Quijote de Cervantes y, a la vez, construir una versión profundamente distinta del original cervantino. Estamos en un nuevo territorio: el ámbito "de la superioridad del lector sobre el autor". Cada lector se convertirá en un nuevo autor del Quijote porque en cada época y lugar se lee con una distinta tradición cultural en cuanto a las representaciones y prácticas de lectura (Rodríguez Fernández, 2005).

Es así como el símil no resulta forzado ante la nueva encrucijada en la cual se encuentran las revistas especializadas: textos cambiantes (con encadenadas aproximaciones en sus cierres) y con diferentes grados de posibles intervenciones de sus creadores o de otros autores o de otros lectores. Pierre Menard, entonces, ha introducido su propio Quijote en la edición de revistas científicas. Un Pierre Menard que ha desembarcado para quedarse junto a nosotros, como susurrándonos al oído, e incrementar su influencia hasta grados que todavía desconocemos.

Pero estos aspectos $\mathrm{u}$ otros cualesquiera requieren de un fundamento ideológico y de una mayor responsabilidad social en la enseñanza de la BCI. Muchas veces los planes de estudio de una carrera deben adaptarse a una realidad que pone en jaque la subsistencia misma de esa rama del saber; pero, en otras oportunidades, hay que meditar los nuevos contenidos ya proyectados en la vanguardia del futuro.

Como una carrera -hablamos en particular de nuestra profesión- dirime su destino por su capacidad de formar una masa crítica y pensante, deviene indispensable crear una materia sobre los Fundamentos y prácticas de Acceso Abierto y, si somos más audaces, implantar una asignatura que pueda abarcar todas estas necesidades -que no son más que derechos: nos referimos ahora a una materia nutricia y en debate constante, bajo la denominación preliminar de Política e ideología en Bibliotecología y Ciencia de la Información, tal como lo manifestamos en otros trabajos. Donde se estudien y discutan cómo toda representación de la Cultura Escrita (y las revistas de Bibliotecología forman parte del pensamiento político) consiste en un campo donde se despliegan las ideologías de dominio y poder, cualesquiera sean. ¿Se puede negar que la circulación de los contenidos rentados y/o comerciales y las realidades del acceso abierto no se articulen con una trazabilidad política sobre la información?

Sin embargo, los retos serán polivalentes y múltiples y, sin duda, con salidas esperadas o inesperadas. Las formas de edición comercial están cambiando en forma tan vertiginosa que la geografía editorial del mundo impreso puede llegar a ser irreconocible en el porvenir. Las condiciones para la autoedición en el cosmos virtual aún no han dicho su última palabra y las iniciativas individuales y grupales pequeñas, tienen una oportunidad de edición y distribución impensadas para la cultura impresa. Pensemos que muchas prácticas de los grandes pools editoriales fueron "tomadas" a partir de gestiones independientes. Y que estos pools abogan por colonizarlas y llevarse, si es posible, toda la torta (con la frutilla y su mejor crema). En este escenario el universo de las revistas académicas debe apropiarse, en toda amplitud, de la totalidad de las prácticas de edición virtual para competir con estos gigantes despiadados en el momento de facturar.

Una batalla, por cierto, digna de David y Goliat, pero no por ello no menos disputable. La evolución de la cultura escrita nos patentiza, una y otra vez, que poco importa el soporte de las palabras, pues siempre se escribirá y leerá lo escrito: lo importante es garantizar un uso social y libre de los textos. Esto introduce otro criterio a tener en cuenta en nuestra disciplina: su facultad de aumentar los fenómenos sociales. Las tecnologías editoriales virtuales pueden alejar a nuestros profesionales de su misión social y autogenerar una especie de BCI aséptica y estéril en sus correspondencias humanas. 
De ahí que la gestación de emprendimientos y acciones de acceso libre aún no pensados, es decir, todavía por crearse, devendrá en otro dilema que debemos imaginar para anclarnos, aún más fuertemente, como un área de las Ciencias Sociales en constante crecimiento.

Al comenzar esta reflexión sobre Información, Cultura y Sociedad en el marco de sus veinte años de existencia, habíamos señalado en un principio que no resultaba satisfactorio un mero balance descriptivo de sus contenidos, pues los próximos veinte años estarán pautados por los dos temas expuestos (tópicos que se encuentran en las antípodas de toda tradición impresa), y que debemos repetirlos nuevamente: la impostergable capacidad de adaptación a las nuevas modalidades virtuales y digitales de edición académica, y la concienciación ampliada del acceso abierto.

Como bibliotecarias y bibliotecarios a cargo de la edición de una revista de BCI tendremos que adaptarnos a estos "novedosos aspectos y categorías" editoriales, y a los cambios que ellas promueven: cambios de autoría, de acceso, de movilidad textual, de formatos, de códigos, de formas y procederes de evaluación, entre otros muchos que hemos comentado. No sea el caso que nos identifiquen con los bibliotecarios decimonónicos que denigró Arthur Rimbaud (1954) en su famoso poema "Los sentados" (Les assis), tal como nos lo advirtió Roberto Juarroz en sus imprescindibles clases hace ya mucho tiempo, donde la falta de iniciativa de nuestros predecesores (sentados, conservadores e inamovibles) fue motivo de una descarnada burla por parte del más joven y maldito poeta francés. Si la reacción es la esperada, acaso también nosotros lleguemos a ser los Pierre Menard de Rimbaud. 


\section{Q Referencias bibliográficas}

》 Black, Bob. 2017. La abolición del trabajo. 2 ed. Mallín Ahogado (Río Negro, Argentina): Estela Eterna.

" CRECS. 2019. Conferencia Internacional sobre revistas científicas en Ciencias Sociales y Humanidades. <http://www.crecs.info/> [Consulta: 24 marzo 2019].

"DORA. 2013. Declaración de San Francisco sobre la evaluación de la Investigación. $<$ https://sfdora.org/wp-content/uploads/2018/og/DORA_Spanish.pdf> [Consulta: 17 marzo 2019].

»El futuro de la publicación académica y la comunicación académica. Unión Europea, enero 2019: Resumen Ejecutivo. 2019. <http://www.editorescientificos. $\mathrm{cl} /$ futuro-publicacion-academica.html $>$ [European Commission. 2019. Future of Scholarly Publishing and Scholarly Communication. Report of the Expert Group to the European Commission. Luxembourg: Publications Office of the European Union. <https://publications.europa.eu/s/klZa> [Consulta: 21 marzo 2019].

" Hicks, Diana; Paul Wouters; Ludo Waltman; Sarah de Rijcke y Ismael Rafols. 2015. Bibliometrics: The Leiden Manifesto for research metrics. En Nature. Vol. 520, no. 7548, 429-431. <https://www.nature.com/news/bibliometrics-theleiden-manifesto-for-research-metrics-1.17351> [Consulta: 22 marzo 2019] [Traducción al español: El manifiesto de Leiden sobre indicadores de investigación. <http://www.ingenio.upv.es/sites/default/files/adjunto-pagina-basica/ manifiesto_es.pdf>

" Illich, Iván. 2008. Obras reunidas II. México: Fondo de Cultura Económica. $<$ https://josefranciscoescribanomaenza.files.wordpress.com/2015/o1/vol-2. pdf> [Consulta: 11 marzo 2019].

»Marx, Karl. 1983 (1842). Los debates sobre la Ley acerca del Robo de Leña. En Defensa de la libertad: los artículos de la Gaceta Renana, 1842-1843. Valencia: Fernando Torres-Editor. p. 204-244.

" McKenzie, Donald F. 2005. Bibliografía y sociología de los textos. Madrid: Akal.

» Revistas científicas Filo [UBA]. 2018. <http://revistascientificas.filo.uba.ar/index. php/ICS> [Consulta: 14 marzo 2019].

》 Rimbaud, Arthur. 1954. Oeuvres complétes. Paris: Librairie Gallimard. p. 70-71. (Bibliothèque de la Pléiade).

"Rodríguez Fernández, Mario. 2005. Pierre Menard, autor del Quijote. Biografía de un lector. En Revista Chilena de Literatura. No. 67, 103-112. <https://scielo. conicyt.cl/scielo.php?script=sci_arttext\&pid=S0718-22952005000200007> [Consulta: 14 marzo 2019]. 
